\title{
Analytic Solutions to a Marcum $Q$-Function-Based Integral and Application in Energy Detection of Unknown Signals over Multipath Fading Channels
}

\author{
Paschalis C. Sofotasios ${ }^{1,2}$, Mikko Valkama ${ }^{1}$, Theodoros A. Tsiftsis ${ }^{2,3}$, Yury A. Brychkov ${ }^{4}$, \\ Steven Freear ${ }^{5}$ and George K. Karagiannidis ${ }^{2,6}$ \\ ${ }^{1}$ Department of Electronics and Communications Engineering, Tampere University of Technology, 33101 Tampere, Finland. \\ e-mail: \{paschalis.sofotasios; mikko.e.valkama\} @tut.fi \\ ${ }^{2}$ Department of Electrical and Computer Engineering, Aristotle University of Thessaloniki, 54124 Thessaloniki, Greece. \\ e-mail: geokarag@ieee.org \\ ${ }^{3}$ Department of Electrical Engineering, Technological Educational Institute of Central Greece, 35100 Lamia, Greece. \\ e-mail: tsiftsis@teilam.gr \\ ${ }^{4}$ The Dorodnicyn Computing Center of the Russian Academy of Sciences, 119333 Moscow, Russia. \\ e-mail: brychkov@ccas.ru \\ ${ }^{5}$ School of Electronic and Electrical Engineering, University of Leeds, LS2 9JT, Leeds, United Kingdom. \\ e-mail: s.freear@leeds.ac.uk \\ ${ }^{6}$ Department of Electrical and Computer Engineering, Khalifa University, PO Box 127788, Abu Dhabi, UAE.
}

\begin{abstract}
This work presents analytic solutions for a useful integral in wireless communications, which involves the Marcum $Q$-function in combination with an exponential function and arbitrary power terms. The derived expressions have a rather simple algebraic representation which renders them convenient both analytically and computationally. Furthermore, they can be useful in wireless communications and particularly in the context of cognitive radio communications and radar systems, where this integral is often encountered. To this end, we derive novel expressions for the probability of detection in energy detection based spectrum sensing over $\eta-\mu$ fading channels. These expressions are given in closed-form and are subsequently employed in analyzing the effects of generalised multipath fading conditions in cognitive radio systems. As expected, it is shown that the detector is highly dependent upon the severity of fading conditions as even slight variation of the fading parameters affect the corresponding performance significantly.
\end{abstract}

\section{INTRODUCTION}

The generalized Marcum $Q$-function, $Q_{m}(a, b)$, is a vital special function in wireless communication theory. It was proposed several decades ago and has appeared extensively in various analyses in the context of stochastic processes in probability theory, single- and multi-channel based communications over fading channels, information-theoretic analysis of multiple-input-multple-output (MIMO) systems, cognitive radio and radar systems, among others [1]-[9], and the references therein. Its use has also led to the derivation of numerous tractable analytic expressions, while its computational realization is rather straightforward since it is included as a built-in function in the most popular software packages [10]-[15].
However, it is widely known that the derivation of tractable analytic expressions in natural sciences and engineering can be rather laborious and cumbersome, if not impossible, as integrals that involve combinations of elementary and special functions are often required to be evaluated analytically [16][21], and the references therein. This is also the case when the Marcum $Q$-function is involved in integrands along with exponential and arbitrary power terms. A general form of such an integral is the following:

$$
\mathcal{I}_{a, b}(k, m, p)=\int_{0}^{\infty} x^{2 k-1} Q_{m}(a x, b) e^{-p x^{2}} d x
$$

which can be equivalently expressed as

$$
\mathcal{I}_{a, b}(k, m, p)=\frac{1}{2} \int_{0}^{\infty} x^{k-1} Q_{m}(a \sqrt{x}, b) e^{-p x} d x .
$$

The integrals in (1) and (2) are encountered in various applications relating to wireless communications, such as in the analysis of multichannel diversity systems with noncoherent and differentially coherent detection and in sensing of unknown signals in the context of cognitive radio and radar systems [22]-[40] and the references therein. Based on this, a recursive formula restricted to only integer values of $k$ and $m$ was reported in [3] while an infinite series representation for the case that $k$ is arbitrary and $m$ is positive integer was recently reported in [41].

Nevertheless, these expressions are neither generic, nor account for the case that $m$ is an arbitrary real. Motivated by this, this work is devoted to the derivation of analytic expressions 
for $\mathcal{I}_{a, b}(k, m, p)$ which can be useful in applications relating to the wide field of digital communications. To this end, novel analytic expressions are derived for the probability of detection of energy detection based spectrum sensing over generalized $\eta-\mu$ fading channels. The derived expressions are given in closed-form and are utilized in analyzing the performance of the detector in various fading conditions.

The remainder of this paper is organized as follows: Sec. II provides the derivation of two analytic expressions for $\mathcal{I}_{a, b}(k, m, p)$. Sec. III is devoted to the application of the offered results in the analytic performance evaluation of energy detection based spectrum sensing over $\eta-\mu$ fading channels for various severity scenarios. The corresponding numerical results are given in Sec. IV along with useful discussions, while closing remarks are provided in Sec. V.

\section{Analytic Solutions to $\mathcal{I}_{a, b}(k, m, p)$ Integrals}

A. A Closed-Form Expression for Arbitrary Integer Values of $k$ and Arbitrary Real Values of $m$

As already mentioned, no analytic expressions for (1) and (2) for the case of arbitrary real values of $m$ have been reported in the open scientific and technical literature.

Theorem 1. For $a, b, m, p \in \mathbb{R}^{+}$and $k \in \mathbb{N}$, the following closed-form expressions is valid,

$$
\begin{aligned}
\mathcal{I}_{a, b}(k, m, p) & =\frac{\Gamma(k) \Gamma\left(m, \frac{b^{2}}{2}\right)}{2 p^{k} \Gamma(m)} \\
& +\sum_{l=0}^{k-1} \frac{a^{2} b^{2 m} \Gamma(k)_{1} F_{1}\left(l+1, m+1, \frac{a^{2} b^{2}}{2 a^{2}+4 p}\right)}{m ! p^{k-l} 2^{m-l+1}\left(a^{2}+2 p\right)^{l+1} e^{\frac{b^{2}}{2}}}
\end{aligned}
$$

where $\Gamma(x)$ and $\Gamma(x, a)$ denote the gamma function and upper incomplete gamma function, respectively, $x ! \triangleq \Gamma(x-1)$ is the increasing factorial and ${ }_{1} F_{1}(x, y, z)$ is the Kummer confluent hypergeometric function [42]-[44].

Proof: By integrating (2) by parts one obtains,

$$
\begin{aligned}
\mathcal{I}_{a, b}(k, m, p) & =\overbrace{\lim _{c \rightarrow \infty}\left[\frac{Q_{m}(a \sqrt{x}, b)}{2} \int x^{k-1} e^{-p x} d x\right]_{0}^{c}}^{\mathcal{T}} \\
& -\frac{1}{2} \int_{0}^{\infty}\left[\int \frac{x^{k-1}}{e^{p x}} d x\right] \frac{d}{d x} Q_{m}(a \sqrt{x}, b) d x
\end{aligned}
$$

where $c$ is a non-negative finite real. By recalling that the lower incomplete gamma function is given by $\gamma(a, x) \triangleq \Gamma(a)-$ $\Gamma(a, x)$, it readily follows that $\int x^{a-1} \exp (-x) d x=\gamma(a, x)=$ $-\Gamma(a, x)$. Upon substituting in (4) one obtains,

$$
\begin{aligned}
\mathcal{T} & =\lim _{c \rightarrow \infty}\left[\frac{Q_{m}(a \sqrt{x}, b) \gamma(k, p x)}{2 p^{k}}\right]_{0}^{c} \\
& =\frac{Q_{m}(0, b) \Gamma(k, 0)}{2 p^{k}}-\lim _{c \rightarrow \infty} \frac{Q_{m}(a \sqrt{c}, b) \Gamma(k, p c)}{2 p^{k}} .
\end{aligned}
$$

With the aid of the identities for $Q_{m}(a, b)$ and $\Gamma(a, x)$ functions in [?], [3] and [42] as well as expressing $d Q_{m}(a, b) / d a$ according to $[3$, eq. (10)], it follows that

$$
\begin{aligned}
\mathcal{I}_{a, b}(k, m, p) & =\sum_{l=0}^{m-1} \frac{\Gamma(k) b^{2 l}}{l ! p^{k} 2^{l}} e^{-\frac{b^{2}}{2}} \\
& +\frac{b^{m}}{p^{k} e^{\frac{b^{2}}{2}}} \int_{0}^{\infty} \frac{\Gamma\left(k, \frac{p y^{2}}{a^{2}}\right) e^{-\frac{y^{2}}{2}} I_{m}(b y)}{y^{m-1}} d y .
\end{aligned}
$$

By making the necessary variable transformation in [43, II. 7 - pp. 726] and substituting in (6) yields

$$
\begin{aligned}
\mathcal{I}_{a, b}(k, m, p) & =\sum_{l=0}^{m-1} \frac{\Gamma(k) b^{2 l}}{l ! p^{k} 2^{l}} e^{-\frac{b^{2}}{2}} \\
& +\sum_{l=0}^{k-1} \frac{b^{m} \Gamma(k) e^{-\frac{b^{2}}{2}}}{l ! p^{k-l} a^{2 l}} \int_{0}^{\infty} \frac{y^{2 l-m+1} I_{m}(b y)}{e^{\frac{y^{2}}{2}\left(1+\frac{2 p}{a^{2}}\right)}} d y .
\end{aligned}
$$

Notably, the above integral can be expressed in closed-form with the aid of [43, eq. (2.15.5.4)]. To this effect, by performing the necessary change of variables and substituting in (7), equation (3) is deduced, which completes the proof.

It is noted that the algebraic representation of the derived solution is simpler than the recursive expression in [3], [4] and additionally, the value of $m$ is subject to no restrictions.

\section{B. An Exact Infinite Series to $\mathcal{I}_{a, b}(k, m, p)$ for Arbitrary Reals}

It is recalled that no analytic expressions exist for (1) and (2) for arbitrary real values, i.e. unrestricted, of all involved parameters.

Lemma 1. For $a, b, k, m, p \in \mathbb{R}^{+}$, the following exact infinite series representation is valid for the integral in (1) and (2),

$$
\mathcal{I}_{a, b}(k, m, p)=\sum_{l=0}^{\infty} \frac{a^{2 l} 2^{k} \Gamma(k+l) \Gamma\left(m+l, \frac{b^{2}}{2}\right)}{l ! \Gamma(m+l)\left(a^{2}+2 p\right)^{k+l}} .
$$

Proof: The $Q_{m}(a, b)$ function can be expressed in infinite series according to [6, eq. (29)]. Therefore, by performing the necessary change of variables it immediately follows that,

$$
Q_{m}(a \sqrt{x}, b)=e^{-\frac{x a^{2}}{2}} \sum_{l=0}^{\infty} \frac{a^{2 l} x^{l} \Gamma\left(l+m, \frac{b^{2}}{2}\right)}{l ! 2^{l} \Gamma(l+m)}
$$

which upon substitution in (2) yields,

$$
\mathcal{I}_{a, b}(k, m, p)=\sum_{l=0}^{\infty} \frac{a^{2 l} \Gamma\left(m+l, \frac{b^{2}}{2}\right)}{l ! 2^{l} \Gamma(m+l)} \underbrace{\int_{0}^{\infty} \frac{x^{k+l-1}}{e^{x\left(p+\frac{a^{2}}{2}\right)}} d x}_{\mathcal{R}} .
$$

The above integral can be expressed in terms of the $\Gamma($. function in [43, eq. (2.10.3.2)], yielding

$$
\mathcal{R}=\frac{2^{k+l} \Gamma(k+l)}{a^{2}+2 p} .
$$

Evidently, by substituting (11) in (10), equation (8) is deduced thus completing the proof. 
The series in (8) is convergent and can provide acceptable accuracy when truncated after relatively few terms. However, deriving a closed-form expression for the truncation error is particularly advantageous in determining the corresponding truncation error accurately and straightforwardly.

Lemma 2. For $a, b, k, m, p \in \mathbb{R}^{+}$, the following inequality can serve as a closed-form upper bound for the truncation error of (8),

$$
\epsilon_{t} \leq \frac{\Gamma(k)}{p^{k}}-\sum_{l=0}^{n} \frac{a^{2 l} 2^{k} \Gamma(k+l) \Gamma\left(m+l, \frac{b^{2}}{2}\right)}{l ! \Gamma(m+l)\left(a^{2}+2 p\right)^{k+l}} .
$$

Proof: The truncation error of (8) when this is truncated after $n$ terms is expressed as,

$$
\begin{aligned}
\epsilon_{t}= & \sum_{l=n+1}^{\infty} \frac{a^{2 l} 2^{k} \Gamma(k+l) \Gamma\left(m+l, \frac{b^{2}}{2}\right)}{l ! \Gamma(m+l)\left(a^{2}+2 p\right)^{k+l}} \\
= & \sum_{l=0}^{\infty} \frac{a^{2 l} 2^{k} \Gamma(k+l) \Gamma\left(m+l, \frac{b^{2}}{2}\right)}{l ! \Gamma(m+l)\left(a^{2}+2 p\right)^{k+l}} \\
& -\sum_{l=0}^{n} \frac{a^{2 l} 2^{k} \Gamma(k+l) \Gamma\left(m+l, \frac{b^{2}}{2}\right)}{l ! \Gamma(m+l)\left(a^{2}+2 p\right)^{k+l}} .
\end{aligned}
$$

It is recalled that the $\Gamma(a, x)$ function is monotonically decreasing w.r.t. $x$ and thus, $\Gamma(a, x) \leq \Gamma(a)$. To this effect, the upper incomplete gamma function in (13) can be bounded as,

$\epsilon_{t} \leq \sum_{l=0}^{\infty} \frac{a^{2 l} 2^{k} \Gamma(k+l)}{l !\left(a^{2}+2 p\right)^{k+l}}-\sum_{l=0}^{n} \frac{a^{2 l} 2^{k} \Gamma(k+l) \Gamma\left(m+l, \frac{b^{2}}{2}\right)}{l ! \Gamma(m+l)\left(a^{2}+2 p\right)^{k+l}}$

By recalling the Pochhammer symbol, $(a)_{n} \triangleq \Gamma(a+n) / \Gamma(a)$, it follows that $\Gamma(k+l)=(k)_{l} \Gamma(k)$. Based on this, the above infinite series can be expressed as follows,

$$
\sum_{l=0}^{\infty} \frac{a^{2 l} 2^{k} \Gamma(k+l)}{l !\left(a^{2}+2 p\right)^{k+l}}=\frac{\Gamma(k) 2^{k}}{\left(a^{2}+2 p\right)^{k}} \sum_{l=0}^{\infty} \frac{a^{2 l}(k)_{l}}{l !\left(a^{2}+2 p\right)^{l}} .
$$

The infinite series in the right-hand side of (15) can be expressed in terms of the hypergeometric function, namely,

$$
\sum_{l=0}^{\infty} \frac{a^{2 l}(k)_{l}(1)_{l}}{l !\left(a^{2}+2 p\right)^{l}(1)_{l}}={ }_{1} F_{0}\left(k ; ; \frac{a^{2}}{a^{2}+2 p}\right)
$$

Based on (16), it immediately follows that

$$
{ }_{1} F_{0}\left(k ; \frac{a^{2}}{a^{2}+2 p}\right)=\frac{\left(a^{2}+2 p\right)^{k}}{2^{k} p^{k}} .
$$

Therefore, by substituting in (17) in (15) one obtains,

$$
\sum_{l=0}^{\infty} \frac{a^{2 l} 2^{k} \Gamma(k+l)}{l !\left(a^{2}+2 p\right)^{k+l}}=\frac{\Gamma(k)}{p^{k}}
$$

Evidently, by substituting (18) in (14) yields (12) thus, completing the proof.

To the best of the Authors' knowledge, equations (3), (8) and (12) have not been previously reported in the open technical literature.

\section{Applications in Cognitive Radio}

\section{A. Energy Detection Based Spectrum Sensing}

Cognitive radio (CR) is an emerging technology that allows opportunistic access of licensed frequency bands when they are not utilized. Given the increased spectrum scarcity along with the high demands for bandwidth resources, CR is anticipated to play a core role in the next generation of mobile communication systems, namely 5G. The most important part of CR technology is the accurate and robust sensing of vacant frequency bands and based on the respective decision the user will decide on whether it can establish communication or not. Therefore, spectrum sensing is the most critical operation in CR systems with energy detection being regarded as the most simple and popular method [23]. In this context, the performance of energy detection based spectrum sensing over various fading conditions have been investigated in [24]-[33], [35] - and the reference therein.

It is recalled that in narrowband energy detection, the received signal waveform follows a binary hypothesis that can be represented as [33, eq. (1)],

$$
r(t)= \begin{cases}n(t) & : H_{0} \\ h s(t)+n(t) & : H_{1}\end{cases}
$$

where $s(t), h$ and $n(t)$ denote an unknown deterministic signal, the amplitude of the channel coefficient and an additive white Gaussian noise (AWGN) process, respectively. The samples of $n(t)$ are assumed to be zero-mean Gaussian random variables with variance $N_{0} W$ with $W$ and $N_{0}$ denoting the single-sided signal bandwidth and a single-sided noise power spectral density, respectively [33]. The hypotheses $H_{0}$ and $H_{1}$ refer to the cases that a signal is absent or present, respectively. The received signal is subject to filtering, squaring and integration over the time interval $T$ which is expressed as [22, eq. (2)], $y \triangleq \frac{2}{N_{0}} \int_{0}^{T}|r(t)|^{2} d t$. The output of the integrator corresponds to a measure of the energy of the received waveform and acts as a test statistic that determines whether the received energy measure corresponds only to the energy of noise $\left(H_{0}\right)$ or to the energy of both the unknown deterministic signal and noise $\left(H_{1}\right)$. By denoting the time bandwidth product as $u=T W$, the test statistic typically follows the central chi-square distribution with $2 u$ degrees of freedom under the $H_{0}$ hypothesis and the non central chisquare distribution with $2 u$ degrees of freedom under the $H_{1}$ hypothesis [24]. Based on this and by recalling that energy detection is largely affected by a predefined energy threshold, $\lambda$, the performance of the detector is characterized by the probability of false alarm, $P_{f}=\operatorname{Pr}\left(y>\lambda \mid H_{0}\right)$ and the probability of detection, $P_{d}=\operatorname{Pr}\left(y>\lambda \mid H_{1}\right)$, namely [22],

$$
P_{f}=\frac{\Gamma\left(u, \frac{\lambda}{2}\right)}{\Gamma(u)}
$$

and

$$
P_{d}=Q_{u}(\sqrt{2 \gamma}, \sqrt{\lambda})
$$

respectively. 


\section{B. The $\eta-\mu$ Distribution}

The $\eta-\mu$ distribution is a generalized fading model that has been widely shown to provide adequate characterization of multipath fading in non-line-of-sight (NLOS) communications. It was reported in [45] along with the $\kappa-\mu$ fading model which accounts for corresponding line-of-sight (LOS) communication scenarios. The $\eta-\mu$ fading model has been shown to be particularly flexible and it includes as special cases the well known Hoyt, Nakagami- $m$, Rayleigh and onesided Gaussian distributions [45]. Its remarkable flexibility and usefulness were demonstrated clearly in [45, Fig. 9] along with the $\kappa-\mu$ fading model where it is clearly shown that the $\eta-\mu$ fading model is significantly more flexible than the more commonly adopted Nakagami $-m$ and Rayleigh distributions.

In terms of physical interpretation, the $\eta-\mu$ fading model is expressed by two physical parameters, $\eta$ and $\mu$ and it holds for two formats, namely Format-1 and Format-2. In the former, the $\eta$ parameter denotes the ratio of the powers between the multipath waves in the in-phase and quadrature components, whereas in the latter it denotes the correlation coefficient between the scattered wave in-phase and quadrature components of each cluster of multipath. Likewise, the $\mu$ parameter denotes - in both formats - the inverse of the normalised variance and relates to the number of multipath clusters in the environment ${ }^{1}$ [45].

The SNR probability density function of the $\eta-\mu$ distribution is expressed as,

$$
p_{\gamma}(\gamma)=\frac{2 \sqrt{\pi} \mu^{\mu+\frac{1}{2}} h^{\mu}}{\Gamma(\mu) H^{\mu-\frac{1}{2}}} \frac{\gamma^{\mu-\frac{1}{2}}}{\bar{\gamma}^{\mu+\frac{1}{2}}} e^{-2 \mu h \frac{\gamma}{\gamma}} I_{\mu-\frac{1}{2}}\left(\frac{2 \mu H \gamma}{\bar{\gamma}}\right)
$$

where $\bar{\gamma}$ denotes the average SNR whereas

$$
h=\frac{2+\eta^{-1}+\eta}{4}, \quad H=\frac{\eta^{-1}-\eta}{4}
$$

in Format-1 with $0<\eta<\infty$ and,

$$
h=\frac{1}{1-\eta^{2}}, \quad H=\frac{\eta}{1-\eta^{2}}
$$

in Format-2 with $-1<\eta<1$. In addition,

$$
\mu=\frac{E^{2}\left(R^{2}\right)}{2 \operatorname{Var}\left(R^{2}\right)}\left[1+\frac{H}{h}\right]
$$

with $E($.$) and \hat{r}$ denoting expectation and the root-meansquare (rms) value of the envelope $R$, respectively [45].

\section{Energy Detection over $\eta-\mu$ Fading Channels}

Corollary 1. For $u, \bar{\gamma}, \lambda \in \mathbb{R}^{+}$and $\mu \in \mathbb{N}$, the following closed-form expressions hold for the average probability of

\footnotetext{
${ }^{1}$ The Format -2 of the $\eta-\mu$ distribution is also known as $\lambda-\mu$ distribution.
}

detection over $\eta-\mu$ fading channels,

$$
\begin{gathered}
\bar{P}_{d}=\sum_{l=0}^{\mu-1} \frac{(\mu)_{l} h^{\mu} \mathcal{G}\left(u, \frac{\lambda}{2}\right)}{l ! 2^{\mu+l} H^{\mu+l}}\left\{\frac{(-1)^{l}}{(h-H)^{\mu-l}}+\frac{(-1)^{\mu}}{(h+H)^{\mu-l}}\right\} \\
+\sum_{l=0}^{\mu-1} \sum_{i=0}^{\mu-l-1} \frac{h^{\mu} \mu^{i} \lambda^{u}(\mu)_{l} \bar{\gamma} e^{-\frac{\lambda}{2}}}{u ! l ! 2^{\mu+u+i-i} H^{\mu+l}} \times \\
\quad\left\{\frac{(-1)^{l}{ }_{1} F_{1}\left(1+i, 1+u, \frac{\lambda \bar{\gamma}}{2 \bar{\gamma}+4 \mu(h-H)}\right)}{(h-H)^{\mu-l-i}(\bar{\gamma}+2(h-H) \mu)^{i+1}}\right. \\
\left.+\frac{(-1)^{\mu}{ }_{1} F_{1}\left(1+i, 1+u, \frac{\lambda \bar{\gamma}}{2 \bar{\gamma}+4 \mu(h+H)}\right)}{(h+H)^{\mu-l-i}(\bar{\gamma}+2(h+H) \mu)^{i+1}}\right\}
\end{gathered}
$$

where $\mathcal{G}(a, x) \triangleq \Gamma(a, x) / \Gamma(a)$ denotes the regularized upper incomplete gamma function and $h$ and $H$ are given by (23) and (24) according to Format-1 and Format-2, respectively.

Proof: The average detection probability is obtained by averaging (21) over the fading statistics of the channel, namely,

$$
\bar{P}_{d}=\int_{0}^{\infty} Q_{u}(\sqrt{2 \gamma}, \sqrt{\lambda}) p_{\gamma}(\gamma) d \gamma .
$$

By substituting (22) in (27) one obtains,

$$
\bar{P}_{d}=\mathcal{A} \int_{0}^{\infty} \frac{Q_{u}(\sqrt{2 \gamma}, \sqrt{\lambda}) I_{\mu-\frac{1}{2}}\left(\frac{2 \mu H \gamma}{\bar{\gamma}}\right)}{\gamma^{\frac{1}{2}-\mu} e^{2 \mu h \frac{\gamma}{\gamma}}} d \gamma
$$

where

$$
\mathcal{A}=\frac{2 \sqrt{\pi} \mu^{\mu+\frac{1}{2}} h^{\mu}}{\Gamma(\mu) H^{\mu-\frac{1}{2}} \gamma^{\mu+\frac{1}{2}}} .
$$

Importantly, for the special case that $\mu$ is a positive integer, the Bessel function in (28) can be expressed in closed-form with the aid of [46, eq. (8.467)] namely,

$$
\begin{aligned}
I_{\mu-\frac{1}{2}}\left(\frac{2 \mu H \gamma}{\bar{\gamma}}\right) & =\sum_{l=0}^{\mu-1} \frac{(-1)^{l} \Gamma(\mu+l) \bar{\gamma}^{l+\frac{1}{2}} e^{\frac{2 \mu H \gamma}{\bar{\gamma}}}}{l ! \sqrt{\pi} \Gamma(\mu-l)(4 \mu H \gamma)^{l+\frac{1}{2}}} \\
& +\sum_{l=0}^{\mu-1} \frac{(-1)^{\mu} \Gamma(\mu+l) \bar{\gamma}^{l+\frac{1}{2}} e^{-\frac{2 \mu H \gamma}{\bar{\gamma}}}}{l ! \sqrt{\pi} \Gamma(\mu-l)(4 \mu H \gamma)^{l+\frac{1}{2}}}
\end{aligned}
$$

Therefore, by substituting (30) in (28) it follows that,

$$
\begin{aligned}
\bar{P}_{d} & =\sum_{l=0}^{\mu-1} \frac{\mathcal{A}(-1)^{l} \Gamma(\mu+l) \bar{\gamma}^{l+\frac{1}{2}}}{l ! \sqrt{\pi} \Gamma(\mu-l)(4 \mu H)^{l+\frac{1}{2}}} \int_{0}^{\infty} \frac{Q_{u}(\sqrt{2 \gamma}, \sqrt{\lambda}) d \gamma}{\gamma^{l-\mu+1} e^{\frac{2 \mu(h-H) \gamma}{\bar{\gamma}}}} \\
& +\sum_{l=0}^{\mu-1} \frac{\mathcal{A}(-1)^{\mu} \Gamma(\mu+l) \bar{\gamma}^{l+\frac{1}{2}}}{l ! \sqrt{\pi} \Gamma(\mu-l)(4 \mu H)^{l+\frac{1}{2}}} \int_{0}^{\infty} \frac{Q_{u}(\sqrt{2 \gamma}, \sqrt{\lambda}) d \gamma}{\gamma^{l-\mu+1} e^{\frac{2 \mu(h+H) \gamma}{\bar{\gamma}}}} .
\end{aligned}
$$

Notably, the integrals in (31) have the same algebraic representation as (1) and (2) and thus, they can be expressed in closedform with the aid of Theorem 1 . As a result, by performing the necessary change of variables in (3), substituting in (31) and carrying out long but basic algebraic manipulations, equation (26) is deduced and thus, the proof is completed. 
Remark 1. The energy threshold in (20) can be expressed as $\lambda=2 \mathcal{G}^{-1}\left(u, P_{f}\right)$, where $\mathcal{G}^{-1}($.$) is the inverse regularized$ upper incomplete gamma function. To this effect (26) can be also equivalently expressed in terms of $P_{f}$ as follows:

$$
\begin{aligned}
& \bar{P}_{d}= \sum_{l=0}^{\mu-1} \frac{(\mu)_{l} h^{\mu} P_{f}}{l ! 2^{\mu+l} H^{\mu+l}}\left\{\frac{(-1)^{l}}{(h-H)^{\mu-l}}+\frac{(-1)^{\mu}}{(h+H)^{\mu-l}}\right\} \\
&+\sum_{l=0}^{\mu-1} \sum_{i=0}^{\mu-l-1} \bar{\gamma} h^{\mu} \mu^{i}\left[\mathcal{G}^{-1}\left(u, P_{f}\right)\right]^{u}(\mu)_{l} \\
& u ! l ! 2^{\mu+i-i} H^{\mu+l} e^{\mathcal{G}^{-1}\left(u, P_{f}\right)} \times \\
& \quad\left\{\frac{(-1)^{l}{ }_{1} F_{1}\left(1+i, 1+u, \frac{\mathcal{G}^{-1}\left(u, P_{f}\right) \bar{\gamma}}{\bar{\gamma}+2 \mu(h-H)}\right)}{(h-H)^{\mu-l-i}(\bar{\gamma}+2(h-H) \mu)^{i+1}}\right. \\
&\left.+\frac{(-1)^{\mu}{ }_{1} F_{1}\left(1+i, 1+u, \frac{\mathcal{G}^{-1}\left(u, P_{f}\right) \bar{\gamma}}{\bar{\gamma}+2 \mu(h+H)}\right)}{(h+H)^{\mu-l-i}(\bar{\gamma}+2(h+H) \mu)^{i+1}}\right\} .
\end{aligned}
$$

To the best of the Authors knowledge (26) and (32) have not been previously reported in the open technical literature.

\section{NUMERICAL RESUltS}

This section is devoted to the analysis of the behaviour of energy detection in $\eta-\mu$ fading conditions by means of $\bar{P}_{d}$ versus $\bar{\gamma}$ curves and complementary receiver operating characteristics (ROC) curves $\left(P_{m}\right.$ versus $\left.P_{f}\right)$. To this end, Fig. 1 illustrates the behavior of the $\bar{P}_{d}$ versus $\bar{\gamma}$ for different values of the fading parameters $\eta$ and $\mu$ for constant time-bandwidth product $u=3$ and the case that $P_{f}=0.01$ and $P_{f}=0.1$. One can notice that the average probability of detection is increased as $\eta$ increases from 0.01 to 0.95 for both cases of $P_{f}$. This is also the case for the $\mu$ parameter as for a fixed value of $\eta$, the $\bar{P}_{d}$ increases when $\mu=3$ compared to the case that $\mu=1$. This also holds for both $P_{f}=0.01$ and $P_{f}=0.1$ and particularly for moderate to high average SNR levels.

In the same context, Fig. 2 depicts the corresponding ROC curves $\left(P_{m}=1-P_{d}\right.$ versus $\left.P_{f}\right)$. The value of $P_{f}$ is assumed between 0.01 and 0.2 while $u=4$ and $\bar{\gamma}=15 \mathrm{~dB}$. One can observe how the performance of the detector improves as the severity of fading is reduced in terms of both $\eta$ and $\mu$. Indicatively, for $P_{f}=0.1$ the value of $P_{m}$ reduces by over $70 \%$ when $\eta$ changes from 0.01 to 0.95 for $\mu=1.0$ and over $65 \%$ when $\mu$ changes from 1.0 to 2.0 for $\eta=0.95$. This demonstrates the sensitivity of the energy detector in multipath fading conditions and how the corresponding severity of fading can affect its performance and robustness.

\section{CONCLUSION}

New expressions were derived for a Marcum $Q$-function based integral that is often encountered in the broad area of digital communications. These expressions include an exact closed-form expression and an infinite series representation along with a closed-form upper bound for the corresponding truncation error. The offered results have a relatively simple algebraic representation which render them analytically and numerically convenient while they can be useful in numerous applications in wireless communications. As an example, they

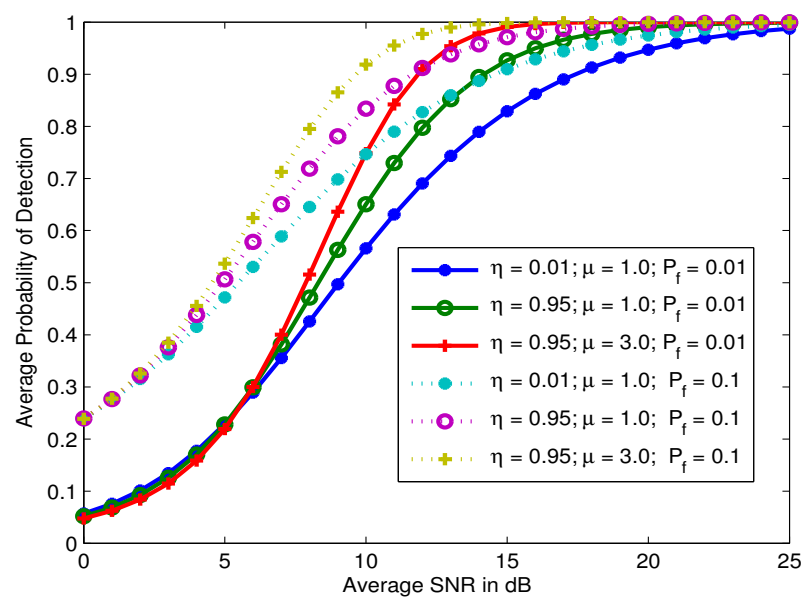

Fig. 1. $\bar{P}_{d}$ vs $\bar{\gamma}$ for different values of $\eta$ and $\mu$ with $u=3$ and $P_{f}=0.01$ and $P_{f}=0.1$.

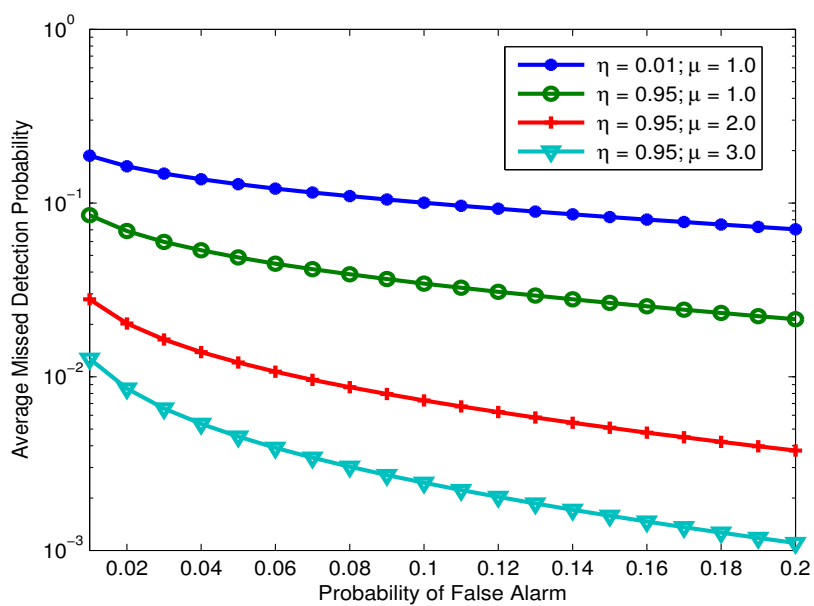

Fig. 2. $\bar{P}_{m}$ vs $P_{f}$ ROC curve for for different values of $\eta$ and $\mu$ with $u=4$ and $\bar{\gamma}=15 \mathrm{~dB}$.

were used in energy detection based spectrum sensing, in the context of cognitive radio and radar systems, deriving novel closed-form expressions for the average probability of detection of unknown signals over $\eta-\mu$ fading channels. The derived expressions were subsequently employed in analyzing the effect of multipath fading on the spectrum sensing performance and it was shown that the overall performance of the detector is, as expected, largely affected by the value of the involved fading parameters, particularly for moderate and high SNR levels.

\section{ACKNOWLEDGEMENTS}

The work of P. C. Sofotasios and M. Valkama was supported by the the Finnish Funding Agency for Technology and Innovation (Tekes) under the project "Enabling Methods for Dynamic Spectrum Access and Cognitive Radio (ENCOR2)" in the TRIAL Program and by the Academy of Finland under the project \#251138 “Digitally-Enhanced RF for Cognitive Radio 
Devices". The work of Yu. A. Brychkov was funded by the Russian Foundation for Fundamental Research under Project No. 13-07-00334. The work of G. K. Karagiannidis was funded in part by the KHALIFA University, under the 2014 KUIRF-Level 1 project: "Revisiting Fundamental Concepts for Green Wireless Communications Networks (REFORM)".

\section{REFERENCES}

[1] J. I. Marcum, "Table of Q-functions, U.S. Air Force Project RAND Res. Memo. M-339, ASTIA document AD 1165451," RAND Corp., Santa Monica, CA, 1950.

[2] P. Swerling, "Probability of detection for fluctuating targets," IRE Trans. on Inf. Theory, vol. IT-6, pp. 269-308, April 1960.

[3] A. H. Nuttall, "Some integrals involving the $Q_{M}$-function," Naval underwater systems center, New London Lab, New London, CT, 1974.

[4] M. K. Simon and M.-S. Alouini, "Some new results for integrals involving the generalized Marcum $Q$-function and their application to performance evaluation over fading channels," IEEE Trans. Wireless Commun., vol. 2, no. 4, July 2003.

[5] M. K. Simon and M.-S. Alouini, "Digital communication over fading channels," Wiley, New York, 2005.

[6] V. M. Kapinas, S. K. Mihos and G. K. Karagiannidis, "On the monotonicity of the generalized Marcum and Nuttall $Q$-functions," IEEE Trans. Inf. Theory, vol. 55, no. 8, pp. 3701-3710, Aug. 2009.

[7] Yu. A. Brychkov, "On some properties of the Marcum $Q$ - function," Integral Transforms and Special Functions, vol. 23, no. 3, pp. 177-182, Mar. 2012.

[8] V. M. Kapinas, Optimization and performance evaluation of digital wireless communication systems with multiple transmit and receive antennas, Ph.D. dissertation, Aristotle University of Thessaloniki, Thessaloniki, Greece, 2014.

[9] Yu. A. Brychkov, "On some properties of the Nuttall function $Q_{\mu, \nu}(a, b), "$ Integral Transforms and Special Functions, vol. 25, no. 1, pp. 34-43, Jan. 2014.

[10] Yu. A. Brychkov, and K. O Geddes, "On the derivatives of the Bessel and Struve functions with respect to the order," Integral Transforms and Special Functions, vol. 16, no. 3, 187-198, Jan. 2007.

[11] A. A. Khan, "Expansions of multivariable Bessel functions," Integral Transforms and Special Functions, vol. 18, no. 7, 481-483, Jul. 2007.

[12] Yu. A. Brychkov, "Power expansions of powers of trigonometric functions and series containing Bernoulli and Euler polynomials," Integral Transforms and Special Functions, vol. 20, no. 11, 797-804, Oct. 2009.

[13] Yu. A. Brychkov, "On multiple sums of special functions," Integral Transforms and Special Functions, vol. 21, no. 12, 797-804, May 2010.

[14] P. C. Sofotasios, S. Freear, "A Novel Representation for the Nuttall $Q$-Function," in Proc. IEEE ICWITS '10, pp. 1-4, Honolulu, HI, USA, August 2010.

[15] Yu. A. Brychkov, "On higher derivatives of the Bessel and related functions," Integral Transforms and Special Functions, vol. 24, no. 8, 607-612, Oct. 2012.

[16] N. Virchenko, S.L. Kalla, and A. Al-Zamel, "Some results on a generalized hypergeometric function," Integral Transforms and Special Functions, vol. 12, no. 1, 89-100, Jan. 2001.

[17] N. O. Virchenko, and V. O. Haidey, "On generalized $m$-bessel functions," Integral Transforms and Special Functions, vol. 8, no. 3-4, 275-286, Apr. 2007.

[18] Yu. A. Brychkov, "Evaluation of Some Classes of Definite and Indefinite Integrals," Integral Transforms and Special Functions, vol. 13, no. 2, 163-167, Oct. 2010.

[19] E. Deniz, "Convexity of integral operators involving generalized Bessel functions," Integral Transforms and Special Functions, vol. 24, no. 3, 201-216, May. 2012.

[20] Yu. A. Brychkov, "Two series containing the Laguerre polynomials," Integral Transforms and Special Functions, vol. 24, no. 11, 911-915, May 2013.

[21] P. C. Sofotasios, T. A. Tsiftsis, Yu. A. Brychkov, S. Freear, M. Valkama, and G. K. Karagiannidis, "Analytic Expressions and Bounds for Special Functions and Applications in Communication Theory," Preprint, ArXIv: 1403.5326, pp. 1-42, Mar. 2014.

[22] F. F. Digham, M. S. Alouini, and M. K. Simon, "On the energy detection of unknown signals over fading channels," IEEE Trans. Commun. vol. 55 , no. 1 , pp. $21-24$, Jan. 2007.
[23] V. K. Bargava, E. Hossain, "Cognitive Wireless Communication Networks," Springer-Verlag, Berlin, Heidelberg 2009.

[24] H. Urkowitz, "Energy detection of unknown deterministic signals," Proc. IEEE, vol. 55, no. 4, pp. 523-531, 1967.

[25] V. I. Kostylev, "Energy detection of signal with random amplitude," in Proc. IEEE Int. Conf. on Communications (ICC '02), pp. 1606-1610, May 2002.

[26] S. P. Herath, N. Rajatheva, C. Tellambura, "On the energy detection of unknown deterministic signal over Nakagami channels with selection combining," in Proc. IEEE Canadian Conf. in Elec. and Comp. Eng. (CCECE' '09), pp. 745-749, May 2009.

[27] A. Ghasemi, E. S. Sousa, "Spectrum sensing in cognitive radio networks: Requirements, challenges and design trade-offs," IEEE Commun. Mag., pp. $32-39$, Apr. 2008.

[28] S. Atapattu, C. Tellambura, H. Jiang, "Energy detection of primary signals over $\eta-\mu$ fading channels," in Proc. $4^{t h}$ Ind. Inf. Systems (ICIIS 09), pp. 1-5, Dec. 2009.

[29] K. Ruttik, K. Koufos and R. Jantti, "Detection of unknown signals in a fading environment," IEEE Comm. Lett., vol. 13, no. 7, pp. 498-500, July 2009 .

[30] P. C. Sofotasios, S. Freear, "Novel expressions for the Marcum and one dimensional $Q$-functions," Proceedings of $7^{\text {th }}$ ISWCS, York, UK, Sep. 2010, pp. 736-740.

[31] P. C. Sofotasios, On special functions and composite statistical distributions and their applications in digital communications over fading channels, Ph.D. Dissertation, University of Leeds, UK, 2010.

[32] S. P. Herath, N. Rajatheva, C. Tellambura, "Energy detection of unknown signals in fading and diversity reception," IEEE Trans. Commun., vol. 59, no. 9, pp. 2443-2453, Sep. 2011.

[33] K. T. Hemachandra, N. C. Beaulieu, "Novel analysis for performance evaluation of energy detection of unknown deterministic signals using dual diversity," in Proc. IEEE Vehicular Tech. Conf. (VTC-fall '11), pp. $1-5$, Sep. 2011

[34] K. Ho-Van, P. C. Sofotasios, "Outage Behaviour of Cooperative Underlay Cognitive Networks with Inaccurate Channel Estimation," in Proc. IEEE ICUFN '13, pp. 501-505, Da Nang, Vietnam, July 2013.

[35] P. C. Sofotasios, E. Rebeiz, L. Zhang, T. A. Tsiftsis, D. Cabric and S. Freear, "Energy detection-based spectrum sensing over $\kappa-\mu$ and $\kappa-\mu$ extreme fading channels," IEEE Trans. Veh. Technol., vol. 63, no 3, pp. 1031-1040, Mar. 2013.

[36] K. Ho-Van, P. C. Sofotasios, "Bit Error Rate of Underlay Multi-hop Cognitive Networks in the Presence of Multipath Fading," in IEEE ICUFN '13, pp. 620-624, Da Nang, Vietnam, July 2013.

[37] P. C. Sofotasios, M. K. Fikadu, K. Ho-Van, M. Valkama, "Energy Detection Sensing of Unknown Signals over Weibull Fading Channels," in Proc. IEEE ATC '13, pp. 414-419, HoChiMing City, Vietnam, Oct. 2013.

[38] K. Ho-Van, P. C. Sofotasios, S. V. Que, T. D. Anh, T. P. Quang, L. P. Hong, "Analytic Performance Evaluation of Underlay Relay Cognitive Networks with Channel Estimation Errors," in Proc. IEEE ATC '13, pp. 631-636, HoChiMing City, Vietnam, Oct. 2013.

[39] K. Ho-Van, P. C. Sofotasios, "Exact BER Analysis of Underlay Decodeand-Forward Multi-hop Cognitive Networks with Estimation Errors," IET Communications, vol. 7, no. 18, pp. 2122-2132, Dec. 2013.

[40] K. Ho-Van, P. C. Sofotasios, S. Freear, "Underlay Cooperative Cognitive Networks, with Imperfect Nakagami- $m$ Fading Channel Information and Strict Transmit Power Constraint," IEEE KICS Journal of Communications and Networks, vol. 16. no. 1, pp. 10-17, Feb. 2014.

[41] G. Cui, L. Kong, X. Yang and D. Ran, "Two useful integrals involving generalised Marcum $Q$-function," Electronic Letters, vol. 48, no. 16, Aug. 2012.

[42] A. P. Prudnikov, Yu. A. Brychkov, and O. I. Marichev, Integrals and Series, 3rd ed. New York: Gordon and Breach Science, vol. 1, Elementary Functions, 1992.

[43] A. P. Prudnikov, Yu. A. Brychkov, O. I. Marichev, Integrals and Series, Gordon and Breach Science Publishers, vol. 2, Special Functions, 1992.

[44] A. P. Prudnikov, Yu. A. Brychkov, O. I. Marichev, Integrals and Series, Gordon and Breach Science Publishers, vol. 3, More Special Functions, 1990.

[45] M. D. Yacoub, "The $\kappa-\mu$ distribution and the $\eta-\mu$ distribution," IEEE Antennas Propag. Mag., vol. 49, no. 1, pp. 68-81, Feb. 2007.

[46] I. S. Gradshteyn and I. M. Ryzhik, Table of Integrals, Series, and Products, in $7^{\text {th }}$ ed. Academic, New York, 2007. 